\title{
A Wide-Field Survey of the Globular Cluster Systems of Giant Galaxies
}

\author{
Katherine L. Rhode \\ Astronomy Department, Wesleyan University, Middletown, CT 06459, USA; \\ kathy@astro.wesleyan.edu
}

Abstract. I present selected results from a wide-field CCD survey of the GC systems of giant galaxies, including showing how measurements of the specific frequency of metal-poor GCs can constrain the redshift of their formation.

\section{Introduction to the Survey}

\subsection{Motivation and Design}

Measurements of the ensemble properties of the globular cluster (GC) systems of massive galaxies can provide a direct test of theories for how such galaxies form. Making these global measurements requires observations of all or most of a galaxy's GC system, which in turn requires wide-field imaging. In recent years Steve Zepf (Michigan State) and I have been collaborating on a widefield CCD survey of the GC systems of giant galaxies. Some motivations for the survey are that: the total numbers of GCs in giant galaxies are uncertain; few spiral galaxies have been studied; and the outer regions of GC systems are largely unexplored, at least by modern CCD studies.

To date we have $B V R$ or $B V I$ imaging of four E/S0 galaxies from the Kitt Peak $4 \mathrm{~m}$ telescope and Mosaic Imager, and nine spiral galaxies from largeformat CCDs on the WIYN $3.5 \mathrm{~m}$ telescope. At the distances of the targets $(10-20 \mathrm{Mpc})$, the GCs are unresolved so we use three-color photometry and good image resolution to select them and to exclude stars and background galaxies. Our aim is to derive reliable global properties of the GC systems and to use them to study galaxy formation and test models for the origin of ellipticals, such as spiral mergers (Ashman \& Zepf 1992), multi-phase collapse (Forbes et al. 1997), and collapse with accretion (Côté et al. 1998).

\subsection{Data and Analysis Methods}

The spiral galaxy images cover 7' x 7' or 10' x 10', depending on the WIYN detector used. This translates to radial coverage of 30-40 kpc. Early-type 
galaxies often have extended GC systems, so the images of the E/S0s are 38' x $38^{\prime}$ and provide radial coverage of $60-120 \mathrm{kpc}$. To find GCs, we create a deep, stacked image in each filter; remove the diffuse galaxy light; find sources above a chosen $\mathrm{S} / \mathrm{N}$ level; discard extended objects; and select point sources with $B V R$ or $B V I$ magnitudes and colors like GCs. We also run completeness tests and fit the GCLF to determine what fraction of GCs are missing given our detection limits. We use Galactic star count models and archival HST data to quantify the contamination that remains from stars and galaxies.

\section{Selected Results}

The survey has so far yielded positions and $B V R$ photometry of hundreds to thousands of GC candidates in eight galaxies. In all cases we have imaged the full radial extent of the systems. The derived properties - e.g., number of GCs, spatial and color distributions, color gradient - are thus global ones and provide an important comparison to model predictions. We find that all the models mentioned in Sect. 1.1 have inconsistencies with the data (see Rhode $\&$ Zepf 2004). Below I highlight two general results from the survey.

\subsection{Total Numbers \& Specific Frequencies}

We calculate the total number of GCs $\left(N_{G C}\right)$ in each galaxy by integrating the derived radial distribution of the GC system from the galaxy center to the radius at which the GC surface density goes to zero within the errors. Table 1 gives $N_{G C}$ and specific frequency $\left(S_{N}\right.$ from Harris \& van den Bergh 1981) for the eight galaxies analyzed. Columns (1)-(3) are galaxy name, type, and magnitude, columns (4)-(5) are $N_{G C}$ and $S_{N}$ from the survey, and columns (6)-(7) list, when applicable, $S_{N}$ from past work and a reference. ( $S_{N}$ from past work combines $N_{G C}$ from the study with the $M_{V}$ we assumed.) Our $S_{N}$ values are $20-75 \%$ lower for four of the six galaxies in Table 1 that were studied previously, and our errors on $S_{N}$ are 2-4 times smaller for all six galaxies. The smaller values are due at least in part to reduced contamination levels. Also, observing the full extent of the GC systems yields smaller, better-constrained total numbers than observing the inner GC system and extrapolating to an arbitrary outer radius, as is typical in past CCD work.

\subsection{Mass-Normalized Number of Blue (Metal-Poor) GCs}

Ashman \& Zepf (1992) proposed that elliptical galaxies form in spiral galaxy mergers and that the metal-poor GCs in ellipticals come directly from the progenitor spirals. If this is true, the galaxy-mass-normalized number of metalpoor GCs (called $T_{\text {blue }}$ ) should be similar for spiral and elliptical galaxies. The well-determined global values from the survey allow us to test this prediction. 
Table 1. Total number and $S_{N}$ for eight survey galaxies

\begin{tabular}{lcrrccr}
\hline Galaxy & Type & $M_{V}$ & $N_{G C}$ & $S_{N}$ & Previous $S_{N}$ & Reference \\
\hline NGC 4472 & E2 & -23.1 & 5900 & $3.6 \pm 0.6$ & $4.5 \pm 1.3$ & Harris 1991 \\
NGC 4406 & E3 & -22.3 & 2900 & $3.5 \pm 0.5$ & $4.6 \pm 1.1$ & Harris 1991 \\
NGC 4594 & S0 & -22.4 & 1900 & $2.1 \pm 0.3$ & $2 \pm 1$ & Bridges \& Hanes 1992 \\
NGC 3379 & E1 & -20.9 & 270 & $1.2 \pm 0.3$ & $1.1 \pm 0.6$ & Harris 1991 \\
NGC 7814 & Sab & -20.4 & 170 & $1.3 \pm 0.4$ & $5.2 \pm 1.7$ & Bothun et al. 1992 \\
NGC 3556 & Sc & -21.2 & 290 & $0.9 \pm 0.4$ & $\ldots$ & $\ldots$ \\
NGC 2683 & Sb & -20.5 & 120 & $0.8 \pm 0.4$ & $2.0 \pm 0.7$ & Harris et al. 1985 \\
NGC 4157 & Sb & -20.4 & 80 & $0.6 \pm 0.3$ & $\ldots$ & $\ldots$ \\
\hline
\end{tabular}

Figure \shows $T_{\text {blue }}$ vs. $\log$ of the stellar mass for eight survey galaxies, plus the Milky Way, M31, and three galaxies from the literature (see Rhode, Zepf, \& Santos 2005 for a list). $T_{\text {blue }}$ is defined as $N_{G C}$ (blue) $/\left(M_{G} / 10^{9} \mathrm{M}_{\odot}\right)$. $T_{\text {blue }}$ for the spiral galaxies is too small to account for the blue GC populations of the giant cluster ellipticals, implying that the merger model cannot explain their formation. The data also show a trend of increasing $T_{\text {blue }}$ with increasing galaxy mass. This is generally consistent with a galaxy and GC system formation scenario described by Santos (2003) that combines biased structure formation with hierarchical merging. In this model, metal-poor GCs form over a finite period in the early Universe, when gas-rich protogalactic fragments merge into larger structures. Structure formation is temporarily suppressed at high- $z$, perhaps due to cosmic reionization. Meanwhile, stellar evolution enriches the intergalactic medium, so any GCs formed later are metal-rich compared to the first generation of GCs. Today's massive galaxies in highdensity regions of the Universe began assembling first, so formed relatively more metal-poor GCs during the initial formation epoch.

Figure 11 shows the expected trend if the formation cutoff for the first generation of GCs occurred at $z=7,11$, or 15 . The curves come from an extended Press-Schechter calculation by G. Bryan (Columbia) that assumes that $T_{\text {blue }}$ is proportional to the fraction of a galaxy's mass that has collapsed into halos of at least $10^{8} \mathrm{M}_{\odot}$ by the truncation redshift. Although a biased, hierarchical scenario appears generally consistent with our $T_{\text {blue }}$ data, more modeling and data are needed to determine whether it can reproduce all of the properties of the galaxy GC systems we observe.

\section{Ongoing and Future Work}

Imaging. We are analyzing WIYN data of more spiral galaxies, have begun using an $8 \mathrm{Kx} 8 \mathrm{~K}$ mosaic $\mathrm{CCD}$ on the MDM-2.4m telescope to image more targets, and also plan to use the WIYN One Degree Imager, which will be commissioned in 2009 and provides 0.5 " resolution over a $1^{\circ}$ field. 


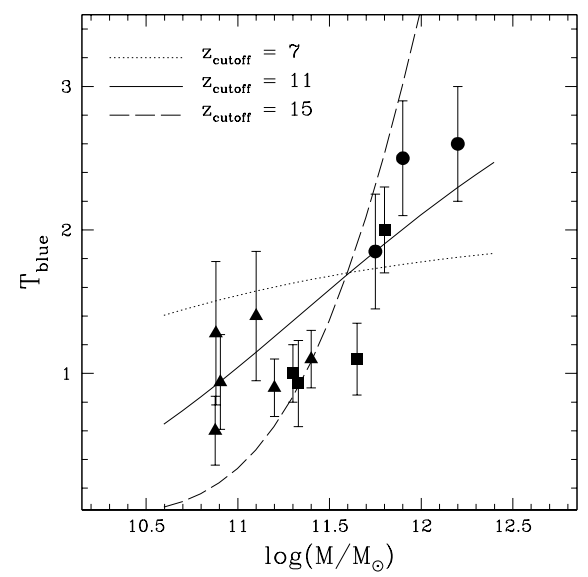

Fig. 1. $T_{\text {blue }}$ vs. log of the galaxy mass for 8 survey galaxies and 5 from the literature. Circles are cluster elliptical galaxies, squares are field E/S0s, and triangles are field spirals. The curves show the expected trend if metal-poor GCs form prior to $z$ of 7 , 11, or 15 (details in Sect. 2.2.

Follow-Up Spectroscopy. With our collaborators, we are using telescopes like the AAT, VLT, Keck, and WIYN to obtain spectra and derive radial velocities of the GC candidates, in order to study the kinematics of the GC systems and measure the mass profiles of the host galaxies to $10-15 R_{\text {eff }}$. Our analysis of the mass distribution of NGC 3379 is published in Bergond et al. 2006.

New Model Predictions. The ideas about blue GCs and biasing in Sect. 2.2 were introduced in Santos (2003) and based on models of the formation of the Milky Way and its GC system. M. Santos (STScI) is modeling the formation of galaxies of varying masses and environments and we are working with him to develop meaningful comparisons between our data and the simulations.

The author is supported by an NSF Astronomy \& Astrophysics Postdoctoral Fellowship under award AST-0302095.

\section{References}

1. Ashman, K.M., \& Zepf, S.E. 1992, ApJ, 384, 50

2. Bergond, G., et al. 2006, A\&A, 448, 155

3. Bothun, G.D., Harris, H.C., \& Hesser, J.E. 1992, PASP, 104, 1220

4. Bridges, T.J. \& Hanes, D.A. 1992, AJ, 103, 800

5. Côté, P., Marzke, R.O., \& West, M.J. 1998, ApJ, 501, 554

6. Forbes, D.A., Brodie, J.P., \& Grillmair, C.J. 1997, AJ, 113, 1652

7. Harris, W.E. 1991, ARAA, 29, 543

8. Harris, W.E. \& van den Bergh, S. 1981, AJ, 86, 1627 
9. Rhode, K.L. \& Zepf, S.E. 2004, AJ, 127, 302

10. Rhode, K.L., Zepf, S.E., \& Santos, M.R. 2005, ApJ, 630, L21

11. Santos, M.R. 2003, in Extragalactic Globular Cluster Systems, ed. M. KisslerPatig (New York: Springer-Verlag), p. 348 